\title{
Ultimate precision of direct tomography of wave functions
}

\author{
Xuan-Hoai Thi Nguyen ${ }^{1} \cdot$ Mahn-Soo Choi $^{1}$ (1) \\ Received: 25 September 2020 / Accepted: 23 June 2021 / Published online: 1 July 2021 \\ (c) The Author(s) 2021
}

\begin{abstract}
In contrast to the standard quantum state tomography, the direct tomography seeks a direct access to the complex values of the wave function at particular positions. Originally put forward as a special case of weak measurement, it has been extended to arbitrary measurement setup. We generalize the idea of "quantum metrology," where a real-valued phase is estimated, to the estimation of complex-valued phase. We show that it enables to identify the optimal measurements and investigate the fundamental precision limit of the direct tomography. We propose a few experimentally feasible examples of direct tomography schemes and, based on the complex phase estimation formalism, demonstrate that direct tomography can reach the Heisenberg limit.
\end{abstract}

Keywords Tomography · Quantum metrology $\cdot$ Heisenberg limit

\section{Introduction}

Reconstruction of the quantum state of a system is of vital importance not only in fundamental studies of quantum mechanics but also in many practical applications of quantum information technology. The standard way to do it, the so-called quantum state tomography, requires an indirect computational reconstruction based on the measurement outcomes of a complete set of non-commuting observables on identically prepared systems [1]. Recently, an alternative method has been put forward and demonstrated experimentally [2,3]. It has attracted much interest because it enables the complex-valued wave functions to be extracted directly and, from many points of view, in an experimentally less challenging manner. We call this method the direct tomography of wave functions. The direct tomography was originally proposed as a special case of weak measurement [2,3]. Later it was extended to arbitrary measurement setup working regardless of the system-pointer coupling strength [4,5]. More recently, the direct tomography has been reinterpreted in the so-called probe-controlled system

\footnotetext{
Mahn-Soo Choi

choims@korea.ac.kr

1 Department of Physics, Korea University, Seoul 02841, South Korea
} 
framework. The latter allows experimenters for even wider variations of setup such as scan-free method of direct tomography [6], and in many cases leads to a higher efficiency. However, the metrological aspects of direct tomography have not been examined at all.

The statistical nature sets the standard quantum limit on the precision of standard measurement techniques $[7,8]$. To reduce the statistical error, one needs to perform a large number $N$ of repeated measurements. When it comes to direct tomography, it seems even worse as the post-selection procedure demands even more repetitions. Recent efforts in quantum metrology have shown new insights to overcome the standard quantum limit and achieve a higher precision by exploiting quantum resources, especially, quantum entanglement [7-10]. A great number of measurement strategies along the line have been proposed and demonstrated experimentally so far [11]. It is known that a genuine multi-particle entanglement is necessary to achieve the maximum precision, the so-called Heisenberg limit [10,12].

In this paper, we investigate the ultimate precision of the direct tomography of wave functions. For the purpose, we generalize the idea of quantum metrology to the estimation of complex-valued phase. We show that the reformulation enables to identify the optimal measurements for efficient estimation and investigate the fundamental precision limit of direct tomography. We further propose two different measurement schemes that eventually approach the Heisenberg limit. In the first method, the pointers are prepared in special entangled states, either GHZ-like maximally entangled state or the symmetric Dicke state. In the other scheme, the ensemble of the measured systems is duplicated and the replica ensemble is time-reversal transformed before the start of the measurement.

Note that here we are concerned with the precision of statistical origin concerning measurement on an ensemble of systems. A notable exception is the so-called protected measurement $[13,14]$, where the expectation value of an observable is obtained from measurement on a single system as demonstrated in a recent experiment [15]. In this case, a higher precision may be achieved by preparing the pointer in a special state of minimum uncertainty such as a squeezed state [16].

\section{Direct tomography as a phase estimation}

In order to investigate the precision limit of the direct tomography of wave functions, it is convenient to reformulate it as a phase estimation. It allows a clearer picture of the optimal initial states and measurements and hence more convenient investigation of the precision limit.

Before the reformulation, we briefly summarize the procedure of direct tomography [2-4]. Here we follow Ref. [4] and examine the direct tomography beyond weakcoupling approximation. Consider an unknown pure state $\left|\psi_{\mathrm{S}}\right\rangle$ in a $d$ dimensional Hilbert space and expand it in a given basis $\{|x\rangle \mid x=1, \cdots, d\}$ as

$$
\left|\psi_{\mathrm{S}}\right\rangle=\sum_{x=1}^{d} \psi_{x}|x\rangle .
$$


A qubit is taken as the pointer and prepared in the state $\left|\phi_{\text {in }}\right\rangle$. The total wave function of the system plus the pointer is thus $\left|\Psi_{\text {in }}\right\rangle=\left|\psi_{S}\right\rangle \otimes\left|\phi_{\text {in }}\right\rangle$. The direct measurement of wave function $\psi_{x}$ starts by coupling the system with the pointer. The system-pointer interaction can be described by a unitary operator of the form

$$
\hat{U}_{x}=\mathrm{e}^{-\mathrm{i} \theta|x\rangle\langle x| \otimes \hat{K} / 2}=\left(\hat{I}_{\mathrm{S}}-|x\rangle\langle x|\right) \otimes \hat{I}_{\mathrm{P}}+|x\rangle\langle x| \otimes \mathrm{e}^{-\mathrm{i} \theta \hat{K} / 2},
$$

where $\theta$ is the system-pointer coupling constant, $\hat{K} / 2$ is a traceless "angular momentum" operator (i.e., $e^{-i \theta \hat{K} / 2}$ is a "rotation" operator) on the pointer, and $\hat{I}_{\mathrm{S}}\left(\hat{I}_{\mathrm{P}}\right)$ is the identity operator on the system (pointer). The initial state $\left|\phi_{\text {in }}\right\rangle$ and the coupling operator $\hat{K}$ of the pointer are chosen such that $\left\langle\phi_{\text {in }}|\hat{K}| \phi_{\text {in }}\right\rangle=0$. Metrologically, it corresponds to the requirement that the pointer rotates as much as possible in response to the system-pointer coupling and enables a higher precision [20]. After the interaction, the system is post-selected on to the state

$$
\left|p_{0}\right\rangle=\frac{1}{\sqrt{d}} \sum_{x=1}^{d}|x\rangle
$$

which leaves the pointer in the state

$$
\left|\phi_{\mathrm{f}}\right\rangle=\frac{1}{\sqrt{|\alpha|^{2}+|\beta|^{2}}}\left(\alpha \hat{I}_{P}-\mathrm{i} \beta \hat{K}\right)\left|\phi_{\text {in }}\right\rangle,
$$

where

$$
\alpha=\tilde{\psi}-\psi_{x}+\psi_{x} \cos (\theta / 2), \quad \beta=\psi_{x} \sin (\theta / 2), \quad \tilde{\psi}=\sum_{x} \psi_{x}
$$

Without loss of generality, $\tilde{\psi}$ is assumed to be real and positive as a global phase factor is physically irrelevant (if $\tilde{\psi}=0$, then one can choose a post-selection to a different state). Now, extracting $\psi_{x}$ is essentially equivalent to the single-qubit quantum state tomography. Accordingly, we choose three observables to measure, $\hat{K} / 2$ in the system-pointer coupling and two other angular momentum operators, $\hat{K}_{1} / 2$ and $\hat{K}_{2} / 2$, perpendicular to $\hat{K} / 2$. Through a number of independent measurements, the probabilities $P_{M}$ for the measurements $M=K, K_{1}, K_{2}$ to yield the outcome 1 (contrary to -1$)$ are inferred, and then the wave function is extracted from the relation

$$
\psi_{x} \propto\left(1-P_{K_{1}}\right) \tan \frac{\theta}{4}+\left(P_{K_{2}}-\frac{1}{2}\right)+\mathrm{i}\left(P_{K}-\frac{1}{2}\right) .
$$

Therefore, in principle, the wave function $\psi_{x}$ is estimated exactly as long as the probabilities $P_{M}$ are inferred out of an infinite number of repeated measurements. In practice, however, the number of repetitions is finite and the accuracy is subject to the standard quantum limit. 
Now let us reformulate the direct tomography outlined above as a phase estimation problem. To this end, we rewrite the normalized pointer state after post-selection into the form

$$
\left|\phi_{\mathrm{f}}\right\rangle=\sqrt{\frac{\alpha^{2}+\beta^{2}}{\left|\alpha^{2}+\beta^{2}\right|}} \frac{\mathrm{e}^{-\mathrm{i} \varphi \hat{K} / 2}\left|\phi_{\text {in }}\right\rangle}{\sqrt{\left\langle\phi_{\text {in }}\left|\mathrm{e}^{\mathrm{i}\left(\varphi^{*}-\varphi\right) \hat{K} / 2}\right| \phi_{\text {in }}\right\rangle}},
$$

where we have introduced a complex-valued phase $\varphi$ by the relations

$$
\cos \frac{\varphi}{2}=\frac{\alpha}{\sqrt{\alpha^{2}+\beta^{2}}}, \quad \sin \frac{\varphi}{2}=\frac{\beta}{\sqrt{\alpha^{2}+\beta^{2}}} .
$$

Once the complex parameter $\varphi$ is estimated through experiments, one can extract the wave function $\psi_{x}$ by

$$
\psi_{x}=\frac{\tilde{\psi} \tan (\varphi / 2)}{\sin (\theta / 2)[1+\tan (\theta / 4) \tan (\varphi / 2)]}
$$

Whereas the relation (7) between the final and initial state is formally the same as the standard phase estimation in quantum metrology [10], it involves two real parameters, $\varphi_{1}:=\operatorname{Re} \varphi$ and $\varphi_{2}:=\operatorname{Im} \varphi$, and corresponds to multi-parameter quantum metrology [17]. Naturally, it requires measurements of more than one observables. Throughout this work, the estimation of complex parameter $\varphi=\varphi_{1}+\mathrm{i} \varphi_{2}$ will be used interchangeably with the multi-parameter estimation of real parameters $\varphi_{1}$ and $\varphi_{2}$.

To see how to estimate the complex phase $\varphi$ in Eq. (7), we note that

$$
\left[\begin{array}{c}
\left\langle\hat{K}_{1}\right\rangle_{\mathrm{f}} \\
\left\langle\hat{K}_{2}\right\rangle_{\mathrm{f}}
\end{array}\right]=\frac{1}{\cosh \left(\varphi_{2}\right)+\sinh \left(\varphi_{2}\right)\langle\hat{K}\rangle_{\text {in }}}\left[\begin{array}{cc}
\cos \left(\varphi_{1}\right) & -\sin \left(\varphi_{1}\right) \\
\sin \left(\varphi_{1}\right) & \cos \left(\varphi_{1}\right)
\end{array}\right]\left[\begin{array}{c}
\left\langle\hat{K}_{1}\right\rangle_{\text {in }} \\
\left\langle\hat{K}_{2}\right\rangle_{\text {in }}
\end{array}\right]
$$

and

$$
\langle\hat{K}\rangle_{\mathrm{f}}=\frac{\sinh \left(\varphi_{2}\right)+\cosh \left(\varphi_{2}\right)\langle\hat{K}\rangle_{\text {in }}}{\cosh \left(\varphi_{2}\right)+\sinh \left(\varphi_{2}\right)\langle\hat{K}\rangle_{\text {in }}}
$$

where $\langle\ldots\rangle_{\mathrm{f}}$ denotes the statistical average $\left\langle\phi_{\mathrm{f}}|\ldots| \phi_{\mathrm{f}}\right\rangle$ and analogously $\langle\ldots\rangle_{\text {in }}$. It is observed from Eqs. (10) and (11) that $\varphi_{1}$ rotates the classical vector $\left(\left\langle\hat{K}_{1}\right\rangle,\left\langle\hat{K}_{2}\right\rangle\right)$ around the axis along $\hat{K}$ whereas $\varphi_{2}$ shifts $\langle\hat{K}\rangle$. Such a rotation angle $\varphi_{1}$ can be estimated by Ramsey-type interferometry whereas the estimation of $\varphi_{2}$ requires an amplitude measurement scheme. In particular, for a choice of $\left|\phi_{\text {in }}\right\rangle$ consistent with the optimal sensitivity such that $\left\langle\hat{K}_{2}\right\rangle_{\text {in }}=\langle\hat{K}\rangle_{\text {in }}=0$, one has

$$
\left\langle\hat{K}_{1}\right\rangle_{\mathrm{f}}=\frac{\cos \left(\varphi_{1}\right)}{\cosh \left(\varphi_{2}\right)}\left\langle\hat{K}_{1}\right\rangle_{\mathrm{in}}, \quad\langle\hat{K}\rangle_{\mathrm{f}}=\tanh \left(\varphi_{2}\right)
$$


In short, the optimal estimation of the complex phase $\varphi$, one needs first to (i) prepare the pointer in the initial state such that $\left\langle\hat{K}_{2}\right\rangle=\langle\hat{K}\rangle=0$, and then (ii) perform measurements of two [rather than three as in Eq. (6)] observables $\hat{K}_{1}$ and $\hat{K}$. Therefore, the reformulation of direct tomography in the form of complex phase estimation is intuitively appealing and helps us find the optimal measurements for efficient estimation.

In passing, we remark that any measurement scheme involving post-selection can essentially be formulated as a complex phase estimation although the estimated phase carries different information depending on the specific measurements. An interesting example is sequential weak measurement [18], which has recently been realized experimentally for two non-commuting observables [19]. In this case, the estimated phase gives the sequential weak values.

\section{Precision limits of the direct tomography}

The estimation of a real-valued phase can reach the Heisenberg limit by exploiting quantum entanglements in the pointers [10]. The question is whether the same limit can be achieved for the estimation of a complex phase involved in the direct tomography. Here we demonstrate that it is indeed possible.

Using N00N state Consider an ensemble of $N$ systems all in the same state $\left|\psi_{S}\right\rangle$. We take a set of $N$ qubits as the pointers and prepare them in the so-called NOON state (or the $N$-qubit GHZ state),

$$
\left|\phi_{\text {in }}\right\rangle=\frac{|0\rangle^{\otimes N}+|1\rangle^{\otimes N}}{\sqrt{2}},
$$

which has proved particularly interesting in high-precision quantum metrology [11]. We couple each system in the ensemble to each corresponding pointer qubit so that the overall unitary operator of the interaction is given by

$$
\hat{U}_{x}^{\otimes N}=\left[\exp \left(-\mathrm{i} \theta|x\rangle\langle x| \otimes \frac{\hat{\sigma}_{z}}{2}\right)\right]^{\otimes N} .
$$

Here we have chosen $\hat{K}=\hat{\sigma}_{z}$ to be concrete. After post-selecting every system on to the state $\left|p_{0}\right\rangle$ in Eq. (3), the (normalized) final state of the pointers is given by

$$
\left|\phi_{\mathrm{f}}\right\rangle_{\mathrm{N} 00 \mathrm{~N}}=\frac{(\alpha-\mathrm{i} \beta)^{N}|0\rangle^{\otimes N}+(\alpha+\mathrm{i} \beta)^{N}|1\rangle^{\otimes N}}{\sqrt{|\alpha-\mathrm{i} \beta|^{2 N}+|\alpha+\mathrm{i} \beta|^{2 N}}}
$$


with $\alpha$ and $\beta$ defined in Eq. (5). Equivalently, in accordance with the phase-estimation formulation in Eq. (7), it can be rewritten as $\left|\phi_{\mathrm{f}}\right\rangle_{\mathrm{N} 00 \mathrm{~N}}$ as follows (up to a global phase):

$$
\left|\phi_{\mathrm{f}}\right\rangle_{\mathrm{N} O 0 \mathrm{~N}}=\frac{\mathrm{e}^{-\mathrm{i} N \varphi / 2}|0\rangle^{\otimes N}+\mathrm{e}^{\mathrm{i} N \varphi / 2}|1\rangle^{\otimes N}}{\sqrt{2 \cosh \left(N \varphi_{2}\right)}} .
$$

Now consider two measurements $\hat{M}_{1}:=\hat{\sigma}_{x}^{\otimes N}$ and $\hat{M}_{2}:=\hat{\sigma}_{z}^{\otimes N}$. We note that

$$
\begin{aligned}
\left\langle\hat{M}_{1}\right\rangle:=\left\langle\hat{\sigma}_{x}^{\otimes N}\right\rangle=\frac{\cos \left(N \varphi_{1}\right)}{\cosh \left(N \varphi_{2}\right)}, \\
\left\langle\hat{M}_{2}\right\rangle:=\left\langle\hat{\sigma}_{z}^{\otimes N}\right\rangle=\tanh \left(N \varphi_{2}\right) \quad(\text { for odd } N) .
\end{aligned}
$$

Assuming small variations of the measurements with the parameter $\varphi=\varphi_{1}+\mathrm{i} \varphi_{2}$, the covariance matrix $C_{i j}(\varphi):=\Delta \varphi_{i} \Delta \varphi_{j}(i, j=1,2)$ of the estimators $\varphi_{1}$ and $\varphi_{2}$ is related to the covariance of the measurement $\left\langle\Delta \hat{M}_{\mu} \Delta \hat{M}_{\nu}\right\rangle(\mu, v=1,2)$ by the error-propagation formula

$$
\left\langle\Delta \hat{M}_{\mu} \Delta \hat{M}_{\nu}\right\rangle=\sum_{i j} \frac{\partial\left\langle\hat{M}_{\mu}\right\rangle}{\partial \varphi_{i}} C_{i j}(\varphi) \frac{\partial\left\langle\hat{M}_{\nu}\right\rangle}{\partial \varphi_{j}} .
$$

Inverting the error propagation formula, we find that the precision is given by

$$
\left(\Delta \varphi_{1}\right)^{2}=\left(\Delta \varphi_{2}\right)^{2}=\frac{\cosh ^{2}\left(N \varphi_{2}\right)}{N^{2}} .
$$

It is concluded that $\hat{M}_{1}$ and $\hat{M}_{2}$ are indeed optimal measurements under the optimal condition $N \varphi_{2} \rightarrow 0$ for the Heisenberg limit. Here we have chosen specific measurements $\hat{M}_{1}$ and $\hat{M}_{2}$, but more general argument in terms of the Fisher information and the Cramer-Rao bound; see "Appendix A".

Using Dicke state Thanks to their experimental relevance, the symmetric Dicke states have also been widely used for quantum entanglement [11]. In particular, it was illustrated that the entanglement in a Dicke state enables one to achieve the Heisenberg-limited interferometry for the single-parameter quantum metrology [20]. Given $N$ qubits, the symmetric Dicke state $|j \equiv N / 2, m\rangle$ with $m=j, j-1, \cdots,-j$ is defined by

$$
|j, m\rangle:=\sqrt{\frac{(j-m) !(j+m) !}{(2 j) !}} \sum_{P} \hat{P} \mid \underbrace{11 \ldots \underbrace{00 \ldots}_{j+m}\rangle,}_{j-m}
$$

where the sum is over all possible permutations $P$ and $\hat{P}$ is the corresponding permutation operator. 
We proceed in a similar manner as with the initial NOON state of pointers. The pointers of $N$ qubits are initially prepared in the particular Dicke state $\left|\phi_{\text {in }}\right\rangle=|j \equiv N / 2,0\rangle$. Each pointer is coupled with a system in the ensemble so that the unitary interaction is given by

$$
\hat{U}_{x}^{\otimes N}=\left[\exp \left(-\mathrm{i} \theta|x\rangle\langle x| \otimes \hat{\sigma}_{y} / 2\right)\right]^{\otimes N}
$$

Here we have chosen $\hat{K}=\hat{\sigma}_{y}$ to make the best use of the characteristic of the Dicke state; namely, the sharp distribution along the equator of the generalized Bloch sphere [11]. After post-selection, the final state (7) of the pointers becomes

$$
\left|\phi_{\mathrm{f}}\right\rangle_{\text {Dicke }}=\frac{\mathrm{e}^{-\mathrm{i} \varphi \hat{J}_{y}}|j, 0\rangle}{\sqrt{\left\langle j, 0\left|\mathrm{e}^{\mathrm{i}\left(\varphi^{*}-\varphi\right) \hat{J}_{y}}\right| j, 0\right\rangle}}
$$

where

$$
\hat{J}_{\mu}=\frac{1}{2} \sum_{k=1}^{N} \hat{\sigma}_{\mu}^{(k)} \quad(\mu=x, y, z) .
$$

For later use, we define the Wigner matrix element

$$
W_{m m^{\prime}}^{(j)}:=\left\langle j, m\left|e^{-i \varphi \hat{J}_{y}}\right| j, m^{\prime}\right\rangle
$$

Here note that the phase $\varphi$ is complex in general. For integer $j$, the expression for the matrix element $W_{m 0}^{(j)}$ is especially simple as

$$
W_{m 0}^{(j)}(\varphi)=P_{j}^{m}(\cos \varphi) \sqrt{\frac{(j-m) !}{(j+m) !}} \quad\left(\varphi_{1}>0\right)
$$

where $P_{j}^{m}(z)$ denotes the associated Legendre polynomial of argument $z$.

Unlike the NOON state, the Dicke state does not allow for simple expressions for the Fisher information and the corresponding Cramer-Rao bound. Instead, we choose the optimal measurements based on the characteristics of the Dicke state and its behavior under the collective rotation $e^{-i \varphi \hat{J}_{y}}$ by a complex angle $\varphi$. As mentioned above, the Dicke state has a sharp distribution along the equator of the Bloch sphere. Then $e^{-i \varphi_{1} \hat{J}_{y}}$ rotates this distribution off the equator. The resulting sharp contrast with the initial state can be detected most efficiently by measuring $\hat{J}_{z}^{2}$. On the other hand, $e^{\varphi_{2} \hat{J}_{y}}$ tends to pull the distribution along the positive $y$-axis. This deviation can be efficiently detected by measuring $\hat{J}_{y}$. Below we demonstrate that $\hat{J}_{y}$ and $\hat{J}_{z}^{2}$ are indeed optimal measurements to achieve the Heisenberg limit. 
We start with the analysis of the measurement of $\hat{J}_{y}$ : By virtue of the theory of angular momentum, we acquire

$$
\begin{aligned}
\left\langle\hat{J}_{y}\right\rangle & =\frac{\mathrm{i} W_{10}^{(j)}\left(2 \mathrm{i} \varphi_{2}\right)}{W_{00}^{(j)}\left(2 \mathrm{i} \varphi_{2}\right)} \sqrt{j(j+1)}, \\
\left\langle\hat{J}_{y}^{2}\right\rangle & =j(j+1)-\frac{\mathrm{i} W_{10}^{(j)}\left(2 \mathrm{i} \varphi_{2}\right)}{W_{00}^{(j)}\left(2 \mathrm{i} \varphi_{2}\right)} \operatorname{coth}\left(2 \varphi_{2}\right) \sqrt{j(j+1)} .
\end{aligned}
$$

It then follows from the error-propagation formula that

$$
\begin{aligned}
\frac{1}{\left(\Delta \varphi_{2}\right)^{2}} & =\frac{1}{\left(\Delta \hat{J}_{y}\right)^{2}}\left(\frac{\partial\left\langle\hat{J}_{y}\right\rangle}{\partial \varphi_{2}}\right)^{2}=4\left(\Delta \hat{J}_{y}\right)^{2} \\
& =4 j(j+1)\left[1-\frac{\mathrm{i} W_{10}^{(j)}\left(2 \mathrm{i} \varphi_{2}\right)}{W_{00}^{(j)}\left(2 \mathrm{i} \varphi_{2}\right)} \frac{\operatorname{coth}\left(2 \varphi_{2}\right)}{\sqrt{j(j+1)}}-\left(\frac{\mathrm{i} W_{10}^{(j)}\left(2 \mathrm{i} \varphi_{2}\right)}{W_{00}^{(j)}\left(2 \mathrm{i} \varphi_{2}\right)}\right)^{2}\right]
\end{aligned}
$$

Equation (29) implies that the larger $\left(\Delta \hat{J}_{y}\right)^{2}$ is the more precise the estimation of $\varphi_{2}$ gets, which leads to the optimal condition $\varphi_{2}=0$. Putting the optimal condition into Eq. (29) gives the Heisenberg limit

$$
\left(\Delta \varphi_{2}\right)_{\mathrm{opt}}^{2}=\frac{2}{N(N+2)}
$$

for the estimation of $\varphi_{2}$. It is interesting to note that the variance $\left(\Delta \varphi_{2}\right)^{2}$ in Eq. (29) depends only on $\varphi_{2}$ but not on $\varphi_{1}$. This is another important feature that allows $\varphi_{2}$ to be estimated independently of $\varphi_{1}$ through the measurement $\hat{J}_{y}$.

To analyze the measurement $\hat{J}_{z}^{2}$ as an estimator of $\varphi_{1}$, we evaluate

$$
\begin{aligned}
\left\langle\hat{J}_{z}^{2}\right\rangle & =\frac{\mathrm{i} W_{10}^{(j)}\left(2 \mathrm{i} \varphi_{2}\right)}{W_{00}^{(j)}\left(2 \mathrm{i} \varphi_{2}\right)} \sqrt{j(j+1)} \sin (\varphi)\left[\operatorname{coth}\left(2 \varphi_{2}\right) \sin (\varphi)-\mathrm{i} \cos (\varphi)\right], \\
\left\langle\hat{J}_{z}^{4}\right\rangle & =\frac{1}{W_{00}^{(j)}\left(2 \mathrm{i} \varphi_{2}\right)} \sum_{m=-j}^{j} m^{4}\left|W_{m 0}^{(j)}(\varphi)\right|^{2} .
\end{aligned}
$$

Unlike the case with the measurement $\hat{J}_{y}$, the moments $\left\langle\hat{J}_{z}^{2}\right\rangle$ and $\left\langle\hat{J}_{z}^{4}\right\rangle$ depend on both $\varphi_{1}$ and $\varphi_{2}$. Therefore one has to use the multi-parameter error-propagation formula (19) with $\hat{M}_{1}=\hat{J}_{z}^{2}$ and $\hat{M}_{2}=\hat{J}_{y}$, which leads to

$$
\left(\Delta \varphi_{1}\right)^{2}=\frac{\left\langle\left(\Delta \hat{J}_{z}^{2}\right)^{2}\right\rangle+\left(\frac{\partial\left\langle\hat{J}_{z}^{2}\right\rangle}{\partial \varphi_{2}}\right)^{2}\left(\Delta \varphi_{2}\right)^{2}-\frac{\partial\left\langle\hat{J}_{z}^{2}\right\rangle}{\partial \varphi_{2}}\left(\frac{\partial\left\langle\hat{J}_{y}\right\rangle}{\partial \varphi_{2}}\right)^{-1}\left\langle\left\{\Delta \hat{J}_{z}^{2}, \Delta \hat{J}_{y}\right\}\right\rangle}{\left(\frac{\partial\left\langle\hat{J}_{z}^{2}\right\rangle}{\partial \varphi_{1}}\right)^{2}},
$$


Fig. 1 The precision, $\left(\Delta \varphi_{1}\right)^{-2}$, as a function $\varphi_{1}$ for different values of $\varphi_{2}$ and $N=50$ qubits in the Dicke state

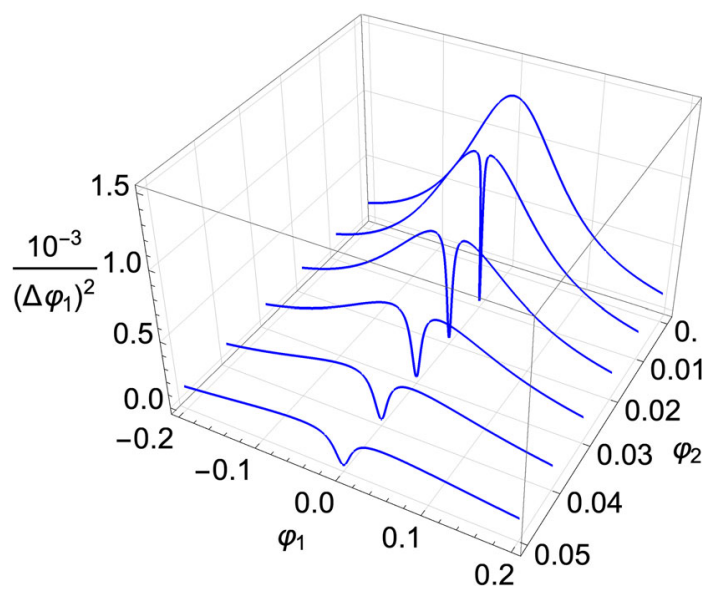

where we have defined $\Delta \hat{A}=\hat{A}-\langle\hat{A}\rangle$ for operator $\hat{A}$ and noted that $\partial\left\langle\hat{J}_{y}\right\rangle / \partial \varphi_{1}=$ 0 . We refer the technical details of its calculations to "Appendix B", and instead summarize its behavior in Fig. 1 as a function of $\varphi_{1}$ and $\varphi_{2}$ for the pointers of $N=50$ qubits. It is clear from Fig. 1 that the optimal condition is given by $\varphi_{1}=\varphi_{2}=0$. At this optimal condition, the precision of $\varphi_{1}$ is given by the Heisenberg limit

$$
\left(\Delta \varphi_{1}\right)_{\mathrm{opt}}^{2}=\frac{2}{N(N+2)}
$$

Incidentally, by putting the optimal condition $\varphi_{2}=0$ obtained independently through the measurement $\hat{J}_{y}$ above, we get

$$
\left.\left(\Delta \varphi_{1}\right)^{-2}\right|_{\varphi_{2}=0}=\frac{8 j(j+1)}{\left(j^{2}+j-2\right) \tan ^{2}\left(\varphi_{1}\right)+4},
$$

which coincides with the single-parameter estimation in Ref. [20] as it should.

Using time-reversal ensemble The reformulation of the direct tomography as a complex phase estimation in Eq. (7) inspires another interesting strategy based on time-reversal (TR) transformation. Given an ensemble of systems in the state in Eq. (1), we prepare another ensemble in the TR state

$$
\left|\bar{\psi}_{\mathrm{S}}\right\rangle:=\hat{T}\left|\psi_{\mathrm{S}}\right\rangle=\sum_{x=1}^{d} \psi_{x}^{*}|x\rangle
$$

where $\hat{T}$ is the (anti-unitary) TR operator (here we assume for simplicity that the basis state $|x\rangle$ is invariant under the TR transformation). The pointers of $2 N$ qubits are prepared, say, in the NOON state. The first $N$ qubits interact with the systems in the original ensemble whereas the other $N$ qubits are coupled with ones in the 
time-reversal ensemble. After post-selection, the pointers get in the final state of the form

$$
\left|\phi_{\mathrm{f}}\right\rangle_{\mathrm{TRS}}=\frac{\left[(\alpha-\mathrm{i} \beta)\left(\alpha^{*}-\mathrm{i} \beta^{*}\right)\right]^{N}|0\rangle^{\otimes 2 N}+\left[\left(\alpha^{*}+\mathrm{i} \beta^{*}\right)(\alpha+\mathrm{i} \beta)\right]^{N}|1\rangle^{\otimes 2 N}}{\sqrt{\left|(\alpha-\mathrm{i} \beta)\left(\alpha^{*}-\mathrm{i} \beta^{*}\right)\right|^{2 N}+\left|\left(\alpha^{*}+\mathrm{i} \beta^{*}\right)(\alpha+\mathrm{i} \beta)\right|^{2 N}}} .
$$

Now recall that for any complex variables $\alpha$ and $\beta$,

$$
\left|(\alpha-\mathrm{i} \beta)\left(\alpha^{*}-\mathrm{i} \beta^{*}\right)\right|=\left|\left(\alpha^{*}+\mathrm{i} \beta^{*}\right)(\alpha+\mathrm{i} \beta)\right| .
$$

It recasts Eq. (37) to the quantum metrologically appealing form

$$
\left|\phi_{\mathrm{f}}\right\rangle_{\mathrm{TRS}}=\frac{|0\rangle^{\otimes 2 N}+\mathrm{e}^{\mathrm{i} 2 N \varphi_{1}}|1\rangle^{\otimes 2 N}}{\sqrt{2}} .
$$

Namely, the above state is identical to the state with the amplified phase shift in interferometries with the NOON state, one of the earliest experimental demonstrations of the Heisenberg limit [21]. It is also worth noting that unlike the above two schemes, in which the estimation of $\varphi_{1}$ strictly depends on that of $\varphi_{2}$, the scheme using the TR ensemble enables the real part $\varphi_{1}$ to be estimated independently. To estimate the imaginary part $\varphi_{2}$, we can apply the measurement strategy proposed in Sect. 3 .

As the TR transformation is anti-unitary, it cannot be implemented physically in isolated systems. However, it is achievable by embedding the system in a larger system. Therefore, as long as the setup permits additional capability of controlling the system, the TR ensemble provides an efficient strategy for direct precision measurement of wave functions.

\section{Conclusion}

Generalizing the idea of quantum metrology of phase estimation, we have reformulated the direct tomography of wave functions as the estimation of complex phase. It has turned out that the new formulation is intuitively appealing and inspires the proper choices of optimal measurements. We have further proposed two different measurement schemes that eventually approach the Heisenberg limit. In the first method, the pointers are prepared in special entangled states, either GHZ-like maximally entangled state or the symmetric Dicke state. In the other scheme, the ensemble of the measured systems is duplicated and the replica ensemble is time-reversal transformed before the start of the measurement. In both methods, the real part of the phase is estimated with Ramsey-type interferometry while the imaginary part is estimated by amplitude measurements. The optimal condition for the ultimate precision is achieved at small values of the complex phases, which provides possible explanations why the previous weak-measurement scheme was successful. The direct tomography relies inevitably on post-selection, and the proposed schemes offer asymptotic gains as the number of pointers in entanglement increases. 
Open Access This article is licensed under a Creative Commons Attribution 4.0 International License, which permits use, sharing, adaptation, distribution and reproduction in any medium or format, as long as you give appropriate credit to the original author(s) and the source, provide a link to the Creative Commons licence, and indicate if changes were made. The images or other third party material in this article are included in the article's Creative Commons licence, unless indicated otherwise in a credit line to the material. If material is not included in the article's Creative Commons licence and your intended use is not permitted by statutory regulation or exceeds the permitted use, you will need to obtain permission directly from the copyright holder. To view a copy of this licence, visit http://creativecommons.org/licenses/by/4.0/.

\section{A The Cramer-Rao bound for the NOON state}

Here we analyze the precision limit of the complex-valued phase estimation based on the multi-parameter estimation in terms of the Fisher information matrix and the corresponding Cramer-Rao bounds [17,22].

We first briefly summarize the multi-parameter quantum metrology [17,23]. Suppose that we want to estimate a set of unknown parameters $\left\{X_{\mu} \mid \mu=1, \cdots, L\right\}$ through the measurements of a positive-operator valued measure (POVM), $\left\{\hat{\Pi}_{j} \mid j=\right.$ $\left.1,2, \ldots, L^{\prime}\right\}$. The covariance matrix $C_{\mu \nu}\left(\left\{X_{\lambda}\right\}\right)=\Delta X_{\mu} \Delta X_{v}$ satisfies the following inequality [17]

$$
C\left(\left\{X_{\mu}\right\}\right) \geq \mathcal{F}^{-1}\left(\left\{\hat{\Pi}_{j}\right\}\right),
$$

where $\mathcal{F}\left(\left\{\Pi_{j}\right\}\right)$ is the Fisher information matrix (FIM) associated with the probability distribution $\left\{p_{j}\left(\left\{X_{\mu}\right\}\right)\right\}$ for the measurements $\left\{\Pi_{j}\right\}$. The entries of the Fisher information matrix are defined by [23]

$$
F_{\mu \nu}=\sum_{j=1}^{L^{\prime}} \frac{\partial_{\mu} p_{j} \partial_{\nu} p_{j}}{p_{j}}
$$

with $\partial_{\mu}$ denoting $\partial / \partial X_{\mu}$. In the case of complex parameters $Z_{\mu}=X_{\mu}+\mathrm{i} Y_{\mu}$, one can keep the complex structure in the covariance matrix and the Fisher information matrix. In this case, one constructs the covariance matrix by replacing each element by the $2 \times 2$ block

$$
C_{\mu \nu}=\left[\begin{array}{lll}
\Delta Z_{\mu} \Delta Z_{v}^{*} \Delta Z_{\mu} \Delta Z_{v} \\
\Delta Z_{\mu}^{*} \Delta Z_{v}^{*} \Delta Z_{\mu}^{*} \Delta Z_{v}
\end{array}\right]
$$

Similarly, the Fisher information matrix is defined with respect to two derivatives $\partial / \partial Z_{\mu}^{*}$ and $\partial / \partial Z_{\mu}$ for each $Z_{\mu}$.

Now let us apply the multi-parameter Cramer-Rao bound (40) in our problem of estimating the wave function $\psi_{x}$ in Eq. (15). Calculating on the final pointer state (15) we obtain the probabilities of the POVM elements as follows:

$$
p_{j}=\left\langle\hat{\Pi}_{j}\right\rangle_{\mathrm{f}, \mathrm{N} 00 \mathrm{~N}}=\frac{A_{j}|\alpha-\mathrm{i} \beta|^{2 N}+B_{j}|\alpha+\mathrm{i} \beta|^{2 N}+2 \operatorname{Re}\left[C_{j}\left(\alpha^{*}+\mathrm{i} \beta^{*}\right)^{N}(\alpha+\mathrm{i} \beta)^{N}\right]}{|\alpha-\mathrm{i} \beta|^{2 N}+|\alpha+\mathrm{i} \beta|^{2 N}},
$$


where

$$
A_{j}=\left\langle 00 \ldots\left|\hat{\Pi}_{j}\right| 00 \ldots\right\rangle, \quad B_{j}=\left\langle 11 \ldots\left|\hat{\Pi}_{j}\right| 11 \ldots\right\rangle, \quad C_{j}=\left\langle 00 \ldots\left|\hat{\Pi}_{j}\right| 11 \ldots\right\rangle
$$

and hence

$$
\partial_{\psi_{x}} p_{j}=N \frac{A_{j}-B_{j}-C_{j}\left(\gamma^{*}\right)^{N}+C_{j}^{*}\left(\gamma^{*}\right)^{-N}}{\left(|\gamma|^{N}+|\gamma|^{-N}\right)^{2}} \partial_{\psi_{x}} \log (\gamma)
$$

with $\gamma=(\alpha-\mathrm{i} \beta) /(\alpha+\mathrm{i} \beta)$. Assuming $|\gamma| \geq 1$ without loss of generality, we see that, as $N \rightarrow \infty, \partial_{\psi_{x}} p_{j} \rightarrow N|\gamma|^{-N}$. As a result, for measurements such that $A_{j}=0$, we find

$$
\left(\mathcal{F}^{-1}\right)_{\mu \nu} \propto \frac{|\gamma|^{N}}{N^{2}}
$$

Therefore, as $|\gamma| \rightarrow 1$, which conforms the optimal condition for the estimation of $\psi_{x}$, the Heisenberg limit is saturated.

\section{B Variance of the real part in the scheme using Dicke state}

In this Appendix we provides the technical details involved in the calculation of the moments $\left\langle\hat{J}_{z}^{2}\right\rangle$ and $\left\langle\hat{J}_{z}^{4}\right\rangle$ in Eqs. (31) and (32), respectively, which are required in Eq. (33).

The terms $\left\langle\hat{J}_{y}\right\rangle, \partial\left\langle\hat{J}_{y}\right\rangle / \partial \varphi_{2}$, and $\left(\Delta \varphi_{2}\right)^{2}$ are given by Eqs. (27) and (29). To calculate the remaining terms in Eq. (33), it is useful to recall the transformation rule

$$
\hat{J}_{\mu}(\varphi)=\mathrm{e}^{\mathrm{i} \varphi \hat{J}_{y}} \hat{J}_{\mu} \mathrm{e}^{-\mathrm{i} \varphi \hat{J}_{y}}=\cos (\varphi) \hat{J}_{\mu}+\mathrm{i}\left[\hat{J}_{y}, \hat{J}_{\mu}\right] \sin (\varphi)
$$

First, let us evaluate the average $\left\langle\hat{J}_{z}^{2}\right\rangle$ and its derivatives. By virtue of Eq. (47), one can obtain

$$
\begin{aligned}
\left\langle\hat{J}_{z}^{2}\right\rangle & =\frac{1}{W_{00}^{(j)}\left(2 \mathrm{i} \varphi_{2}\right)}\left\langle j, 0\left|\mathrm{e}^{\mathrm{i} \varphi^{*} \hat{J}_{y}} \hat{J}_{z}^{2} \mathrm{e}^{-\mathrm{i} \varphi \hat{J}_{y}}\right| j, 0\right\rangle \\
& =\frac{1}{W_{00}^{(j)}\left(2 \mathrm{i} \varphi_{2}\right)} \sum_{m=-j}^{j}\left\langle j, 0\left|\mathrm{e}^{\mathrm{i} 2 \varphi_{2} \hat{J}_{y}}\right| j, m\right\rangle\left\langle j, m\left|\mathrm{e}^{\mathrm{i} \varphi \hat{J}_{y}} \hat{J}_{z}^{2} \mathrm{e}^{-\mathrm{i} \varphi \hat{J}_{y}}\right| j, 0\right\rangle \\
& =\sum_{m=-j}^{j} \frac{W_{0 m}^{(j)}\left(2 \mathrm{i} \varphi_{2}\right)}{W_{00}^{(j)}\left(2 \mathrm{i} \varphi_{2}\right)}\left[\sin ^{2} \varphi\left\langle j, m\left|\hat{J}_{x}^{2}\right| j, 0\right\rangle-\frac{\sin (2 \varphi)}{2}\left\langle j, m\left|\hat{J}_{z} \hat{J}_{x}\right| j, 0\right\rangle\right] \\
& =\frac{\mathrm{i} W_{10}^{(j)}\left(2 \mathrm{i} \varphi_{2}\right)}{W_{00}^{(j)}\left(2 \mathrm{i} \varphi_{2}\right)} \sqrt{j(j+1)} \sin (\varphi)\left[\operatorname{coth}\left(2 \varphi_{2}\right) \sin (\varphi)-\mathrm{i} \cos (\varphi)\right]
\end{aligned}
$$


Noting that

$$
\begin{aligned}
& {\left[\sqrt{\frac{(j+2) !}{(j-2) !}} \frac{W_{20}^{(j)}\left(2 \mathrm{i} \varphi_{2}\right)}{W_{00}^{(j)}\left(2 \mathrm{i} \varphi_{2}\right)}+j(j+1)\right] \sin \left(\mathrm{i} 2 \varphi_{2}\right)} \\
& +2 \sqrt{\frac{(j+1) !}{(j-1) !}} \frac{W_{10}^{(j)}\left(2 \mathrm{i} \varphi_{2}\right)}{W_{00}^{(j)}\left(2 \mathrm{i} \varphi_{2}\right)} \cos \left(\mathrm{i} 2 \varphi_{2}\right)=0,
\end{aligned}
$$

which is derived from the recurrence formula of the associated Legendre polynomial, $\left\langle\hat{J}_{z}^{2}\right\rangle$ can be reduced to Eq. (31). Taking the derivative of $\left\langle\hat{J}_{z}^{2}\right\rangle$ given by Eq. (31) with respect to $\varphi_{1}$ and $\varphi_{2}$, respectively, we obtain

$$
\begin{aligned}
\frac{\partial\left\langle\hat{J}_{z}^{2}\right\rangle}{\partial \varphi_{1}}= & \frac{2 \sin \left(2 \varphi_{1}\right)}{\sinh \left(\varphi_{2}\right)} \frac{\mathrm{i} W_{20}^{(j)}\left(2 \mathrm{i} \varphi_{2}\right)}{W_{00}^{(j)}\left(2 \mathrm{i} \varphi_{2}\right)} \sqrt{j(j+1)} \\
\frac{\partial\left\langle\hat{J}_{z}^{2}\right\rangle}{\partial \varphi_{2}}= & j(j+1)\left[\operatorname{coth}\left(2 \varphi_{2}\right)-\frac{\cos \left(2 \varphi_{1}\right)}{\sinh \left(2 \varphi_{2}\right)}\right]\left[1-\left(\frac{\mathrm{i} W_{20}^{(j)}\left(2 \mathrm{i} \varphi_{2}\right)}{W_{00}^{(j)}\left(2 \mathrm{i} \varphi_{2}\right)}\right)^{2}\right] \\
& +\frac{\mathrm{i} W_{20}^{(j)}\left(2 \mathrm{i} \varphi_{2}\right)}{W_{00}^{(j)}\left(2 \mathrm{i} \varphi_{2}\right)} \sqrt{j(j+1)}\left[\frac{2 \cos \left(2 \varphi_{1}\right) \cosh \left(2 \varphi_{2}\right)-2}{\sinh ^{2}\left(2 \varphi_{2}\right)}-1\right] .
\end{aligned}
$$

On the other hand, $\left\langle\hat{J}_{z}^{4}\right\rangle$ can be expressed in terms of the Wigner matrix elements as following

$$
\begin{aligned}
\left\langle\hat{J}_{z}^{4}\right\rangle & =\frac{1}{W_{00}^{(j)}\left(2 \mathrm{i} \varphi_{2}\right)}\left\langle j, 0\left|\mathrm{e}^{\mathrm{i} \varphi^{*} \hat{J}_{y}} \hat{J}_{z}^{4} \mathrm{e}^{-\mathrm{i} \varphi \hat{J}_{y}}\right| j, 0\right\rangle \\
& =\frac{1}{W_{00}^{(j)}\left(2 \mathrm{i} \varphi_{2}\right)} \sum_{m, m^{\prime}} W_{m^{\prime} 0}^{(j)}\left(\varphi^{*}\right) W_{m 0}^{(j)}(\varphi)\left\langle j, m^{\prime}\left|\hat{J}_{z}^{4}\right| j, m\right\rangle \\
& =\frac{1}{W_{00}^{(j)}\left(2 \mathrm{i} \varphi_{2}\right)} \sum_{m=-j}^{j} m^{4}\left|W_{m 0}^{(j)}(\varphi)\right|^{2} .
\end{aligned}
$$

\section{References}

1. Lvovsky, A.I., Raymer, M.G.: Continuous-variable optical quantum-state tomography. Rev. Mod. Phys. 81(1), 299 (2009). https://doi.org/10.1103/revmodphys.81.299

2. Lundeen, J.S., Sutherland, B., Patel, A., Stewart, C., Bamber, C.: Direct measurement of the quantum wavefunction. Nature 474(7350), 188 (2011). https://doi.org/10.1038/nature 10120

3. Lundeen, J.S., Bamber, C.: Procedure for direct measurement of general quantum states using weak measurement. Phys. Rev. Lett. 108(7), 070402 (2012). https://doi.org/10.1103/physrevlett.108.070402

4. Vallone, G., Dequal, D.: Strong measurements give a better direct measurement of the quantum wave function. Phys. Rev. Lett. 116(4), 040502 (2016). https://doi.org/10.1103/physrevlett.116.040502

5. Calderaro, L., Foletto, G., Dequal, D., Villoresi, P., Vallone, G.: Direct reconstruction of the quantum density matrix by strong measurements. Phys. Rev. Lett. (2018). https://doi.org/10.1103/physrevlett. 121.230501 
6. Ogawa, K., Yasuhiko, O., Kobayashi, H., Nakanishi, T., Tomita, A.: A framework for measuring weak values without weak interactions and its diagrammatic representation. New J. Phys. 21(4), 043013 (2019). https://doi.org/10.1088/1367-2630/ab0773

7. Braunstein, S.L., Caves, C.M.: Statistical distance and the geometry of quantum states. Phys. Rev. Lett. 72, 3439 (1994). https://doi.org/10.1103/PhysRevLett.72.3439

8. Braunstein, S.L., Caves, C.M., Milburn, G.: Generalized uncertainty relations: theory, examples, and Lorentz invariance. Ann. Phys. 247(1), 135 (1996). https://doi.org/10.1006/aphy.1996.0040

9. Giovannetti, V.: Quantum-enhanced measurements: beating the standard quantum limit. Science 306(5700), 1330 (2004). https://doi.org/10.1126/science.1104149

10. Giovannetti, V., Lloyd, S., Maccone, L.: Quantum metrology. Phys. Rev. Lett. 96(1), 010401 (2006). https://doi.org/10.1103/PhysRevLett.96.010401

11. Pezzè, L., Smerzi, A., Oberthaler, M.K., Schmied, R., Treutlein, P.: Quantum metrology with nonclassical states of atomic ensembles. Rev. Mod. Phys. 90(3), 035005 (2018). https://doi.org/10.1103/ revmodphys.90.035005

12. Pezze, L., Smerzi, A.: Entanglement, nonlinear dynamics, and the Heisenberg limit. New J. Phys. 102, 100401 (2009)

13. Aharonov, Y., Vaidman, L.: Measurement of the Schrödinger wave of a single particle. Phys. Lett. A 178(1-2), 38 (1993). https://doi.org/10.1016/0375-9601(93)90724-e

14. Aharonov, Y., Anandan, J., Vaidman, L.: Meaning of the wave function. Phys. Rev. A 47(6), 4616 (1993)

15. Piacentini, F., Avella, A., Rebufello, E., Lussana, R., Villa, F., Tosi, A., Gramegna, M., Brida, G., Cohen, E., Vaidman, L., Degiovanni, I.P., Genovese, M.: Determining the quantum expectation value by measuring a single photon. Nat. Phys. 13(12), 1191 (2017). https://doi.org/10.1038/nphys4223

16. Caves, C.M.: Quantum-mechanical noise in an interferometer. Phys. Rev. D 23, 1693 (1981)

17. Szczykulska, M., Baumgratz, T., Datta, A.: Multi-parameter quantum metrology. Adv. Phys. X 1(4), 621 (2016). https://doi.org/10.1080/23746149.2016.1230476

18. Mitchison, G., Jozsa, R., Popescu, S.: Sequential weak measurement. Phys. Rev. A 76(6), 062105 (2007). https://doi.org/10.1103/physreva.76.062105

19. Piacentini, F., Avella, A., Levi, M., Gramegna, M., Brida, G., Degiovanni, I., Cohen, E., Lussana, R., Villa, F., Tosi, A., Zappa, F., Genovese, M.: Measuring incompatible observables by exploiting sequential weak values. Phys. Rev. Lett. 117(17), 170402 (2016). https://doi.org/10.1103/physrevlett. 117.170402

20. Apellaniz, I., Lücke, B., Peise, J., Klempt, C., Tóth, G.: Detecting metrologically useful entanglement in the vicinity of Dicke states. New J. Phys. 17(8), 083027 (2015). https://doi.org/10.1088/1367-2630/ $17 / 8 / 083027$

21. Bollinger, J.J., Itano, W.M., Wineland, D.J., Heinzen, D.J.: Optimal frequency measurements with maximally correlated states. Phys. Rev. A 54(6), R4649 (1996). https://doi.org/10.1103/physreva.54. r4649

22. Holevo, A.S.: Probabilistic and Statistical Aspects of Quantum Theory. North Holland Publishing Co., Amsterdam (1982). https://doi.org/10.1007/978-88-7642-378-9

23. Hradil, Z., Řeháček, J., Fiurášek, J., Ježek, M.: Maximum-likelihood methodsin quantum mechanics. In: Paris, M., Řeháček, J. (eds) Quantum State Estimation. Lecture Notes in Physics, vol. 649, chap. 3, pp. 59-112. Springer, Berlin (2004). https://doi.org/10.1007/978-3-540-44481-7_3

Publisher's Note Springer Nature remains neutral with regard to jurisdictional claims in published maps and institutional affiliations. 